\title{
Optimization of multi-response dynamic systems using principal component analysis (PCA)- based utility theory approach
}

\section{Susanta Kumar Gauri*}

SQC \& OR Unit, Indian Statistical Institute, 203, B. T. Road, Kolkata-700108, India

\begin{tabular}{l}
\hline C H R O N I C L E \\
\hline Article history: \\
Received July 22013 \\
Received in revised format \\
September 72013 \\
Accepted September 122013 \\
Available online \\
September 142013 \\
\hline Keywords: \\
Dynamic system \\
Multiple responses \\
Optimization \\
Principal component analysis \\
Utility theory \\
\hline
\end{tabular}
A B S T R A C T

\begin{abstract}
Optimization of a multi-response dynamic system aims at finding out a setting combination of input controllable factors that would result in optimum values for all response variables at all signal levels. In real life situation, often the multiple responses are found to be correlated. The main advantage of PCA-based approaches is that it takes into account the correlation among the multiple responses. Two PCA-based approaches that are commonly used for optimization of multiple responses in dynamic system are PCA-based technique for order preference by similarity to ideal solution (TOPSIS) and PCA-based multiple criteria evaluation of the grey relational model (MCE-GRM). This paper presents a new PCA-based approach, called PCA-based utility theory (UT) approach, for optimization of multiple dynamic responses and compares its optimization performance with other existing PCA-based approaches. The results show that the proposed PCA-based UT method is superior to the other PCA-based approaches.
\end{abstract}

(C) 2013 Growing Science Ltd. All rights reserved

\section{Introduction}

The usefulness of Taguchi method (Taguchi, 1990) in optimizing the parameter design in static as well as dynamic system has been well established. In a static system, the response variable representing the output quality characteristic of the system has a fixed target value. A dynamic system differs from a static system in that it contains signal factor and the target value depends on the level of the signal factor set by the system operator. For example, signal factor may be the steering angle in the steering mechanism of an automobile or the speed control setting of a fan. In other words, a dynamic system has multiple target values of the response variables depending on the setting of signal variable of the system.

Optimization of multiple responses in static system has drawn maximum attention of the researchers (Derringer \& Suich, 1980; Khuri \& Conlon, 1981; Pignatiello, 1993; Su \& Tong, 1997; Wu \& * Corresponding author. Tel.: 091-033-2575-5951, Fax: 091-033-2577-6042

E-mail: susantagauri@hotmail.com (S.K. Gauri) 
Hamada, 2000; Tong \& Hsieh, 2001; Wu, 2005; Liao, 2006; Kim \& Lee, 2006; Tong et al., 2007; Jeong \& Kim, 2009; Pal \& Gauri, 2010 a 2010 b. Product/process design with a dynamic system offers the flexibility needed to satisfy customer requirements and can enhance a manufacturer's competitiveness. In recent time, therefore, many researchers have been motivated to study the robust design problem concerning the dynamic systems. Miller and $\mathrm{Wu}(1996)$ have observed that Taguchi's dynamic signal-to-noise ratio (SNR) is appropriate for certain measurement systems but not for multiple target systems. Wasserman (1996) has observed that the factor-level combination of a dynamic system using Taguchi's SNR might not be optimal. McCaskey and Tsui (1997) have found that Taguchi's procedure for dynamic system is appropriate only under a multiplicative model. Lunani et al. (1997) have noted that using SNR as a quality performance measure might produce inaccuracies due to a biased dispersion effect, thus making it impossible to minimize quality loss. Tsui (1999) investigated the direct application of the response model (RM) approach for the dynamic robust design problem. Joseph and $\mathrm{Wu}$ (2002) formulated the robust parameter design of dynamic system as a mathematical programming problem. Chen (2003) developed a stochastic optimization modeling procedure that incorporated a sequential quadratic programming technique to determine the optimal factor-level combination in a dynamic system. Lesperance and Park (2003) have proposed the use of a joint generalized linear model (GLM) so that model assumptions can be investigated using residual analysis. Su et al. (2005) have proposed a hybrid procedure combining neural networks and scatter search to optimize the continuous parameter design problem. Bae and Tsui (2006) have generalized Tsui's (1999) RM approach based on a GLM and reported that the GLM-RM approach can provide more reliable results. It may be noted that all these research articles are focused on optimization of a single-response dynamic system.

Industry has increasingly emphasized developing procedures capable of simultaneously optimizing the dynamic multi-response problems in light of the increasing complexity of modern product design. To cope with the need of the modern industries, several studies (Tong et al., 2002; Hsieh et al., 2005; Wu, 2009; Chang, 2006; Chang, 2008; Tong et al., 2008; Chang and Chen, 2011, Tong et al., 2004; Wang, 2007) have recommended procedures for optimizing multiple responses in a dynamic system. The various approaches for solving multi-response optimization problems in dynamic system can broadly be classified into three categories, e.g. (1) Response surface methodology and desirability function (RSM-DF) based approaches (Tong et al., 2002; Hsieh et al., 2005; Wu, 2009) (2) Artificial intelligence (AI) based approaches (Chang, 2006; Chang, 2008; Tong et al., 2008; Chang and Chen, 2011 ) and (3) Principal component analysis (PCA) based approaches (Tong et al., 2004; Wang, 2007). The basic advantage of using desirability function as performance metric is that it is a simple unitless measure and can allow the user to weigh the responses according to their importance. A disadvantage with this metric is that it does not consider the expected variability and thus the obtained solution may not yield an ideal result. The AI based approaches uses the techniques of artificial neural network (ANN) and genetic algorithm (GA) to solve multi-response optimization problems. The advantage of AI-based technique is that it does not require any specific relationship between quality characteristics and signal factor. The main disadvantage with AI-based approaches is that the information it contains is implicit and virtually inaccessible to the user. So the engineers cannot obtain efficient engineering information during the period of the optimization process.

In real life situation, often the multiple responses are found to be correlated. The main advantage of PCA-based approaches is that it takes into account correlation among the multiple responses. Tong et al. (2004) have proposed a PCA-based technique for order preference by similarity to ideal solution (TOPSIS) method, whereas Wang (2007) has proposed a PCA-based multiple criteria evaluation of the grey relational model (MCE-GRM) for optimization of multiple responses in a dynamic system. The PCA-based approaches are easily understandable and can be implemented using Excel sheet. So this approach has gained quite popularity among the practitioners. This paper presents a new PCA-based approach for optimization of multiple dynamic responses, called PCA-based utility theory (UT) approach and compares its optimization performance with other existing PCA-based approaches. The 
results show that the proposed PCA-based UT method is very promising for optimization of multiresponse dynamic systems.

This article is organized as follows: the second section outlines briefly the dynamic system and the generic approach for application of PCA-based methods for optimizing multi-response dynamic systems. The third section describes the utility concept and the proposed PCA-based UT method for optimizing multiple dynamic responses. In the next section, analyses of two experimental data sets taken from literature are presented. We conclude in the final section.

\section{Dynamic system and the PCA-based approaches for multi-response optimization}

For dynamic system, ideal quality is based on the ideal relationship between the signal and response, and quality loss is caused by deviations from the ideal relationship. So, significant quality improvement can be achieved by first defining a system's ideal function, then using designed experiments to search for an optimal design which minimizes deviations from this ideal function. A dynamic system generally assumes that a linear form exists between the response and the signal factor. The ideal function can be expressed as follows:

$Y=\beta M+\varepsilon$,

where $Y$ denotes the response of a dynamic system, $M$ represents the signal factor, $\beta$ is the slope and $\varepsilon$ denotes the random error. Here, $\varepsilon$ is assumed to follow a normal distribution with a mean of zero and variance of $\sigma^{2}$. The deviation from the ideal function is represented by the variability of the dynamic system $\left(\sigma^{2}\right)$. The objective is to determine the best combination of input controllable variables so that the system achieves the respective target value at each signal factor level with minimum variability around the target value.

Let, $y_{k l}$ denotes the value of the response variable $Y$ at the combination of $k^{\text {th }}$ level of signal factor $\left(M_{k}\right.$ ) $(k=1,2, \ldots, s)$ and $l^{\text {th }}$ level of noise factor $\left(N_{l}\right)(l=1,2, \ldots, n)$. Then, the slope $\beta$ and variability $\sigma^{2}$ of a single response dynamic system can be respectively obtained using the following equations (Taguchi, 1990):

$$
\begin{aligned}
& \beta=\frac{\sum_{k=1 l=1}^{s} \sum_{l=1}^{n} y_{k l} M_{k}}{\sum_{k=1 l=1}^{s} \sum_{k}^{n} M_{k}^{2}} \\
& \sigma^{2}=\frac{1}{s n-1} \sum_{k=1 l=1}^{s} \sum_{k}^{n}\left(y_{k l}-\beta M_{k}\right)^{2}
\end{aligned}
$$

Taguchi used SNR $(\eta)$ and system sensitivity $(S S)$ as the performance measures in a dynamic system to assess the robustness of a process (Tong et al, 2004; Wang, 2007). The SNR and SS values for $j^{\text {th }}$ response variable corresponding to $i^{\text {th }}$ trial, $\eta_{i j}$ and $S S_{i j}$, can be obtained using the following equations:

$$
\begin{gathered}
\eta_{i j}=10 \log _{10}\left(\frac{\beta_{i j}^{2}}{\sigma_{i j}^{2}}\right) \\
S S_{i j}=10 \log _{10} \beta_{i j}^{2}
\end{gathered}
$$

where $\beta_{i j}$ and $\sigma_{i j}^{2}$ are the estimates of the slope and variance of the ideal function for $j^{\text {th }}(j=1,2, \ldots, p)$ response variable corresponding to $i^{\text {th }}(i=1,2, \ldots, m)$ trial. The PCA-based approaches for optimizing multi-response dynamic system broadly use the following three steps: 
Step 1: Converting SNR values of the multiple responses into an overall SNR index (SNRI) and converting SS values of the multiple responses into an overall SS index (SSI) taking into account the correlation among the SRN values and SS values respectively.

Step 2: Determining the significant/influencing factors with respect to SNRI and SSI values. Then, obtaining the optimal factor-level combination that optimizes SNRI value, and identifying the adjustment factor (i.e. the factor that has a large effect on the SSI but no effect on SNRI).

Step 3: Changing the level of the adjustment factor (if available) in the chosen optimal factor-level combination in such a way that the expected output values of the response variables becomes closer to their target values.

The two PCA-based methods (Tong et al., 2004; Wang, 2007) mainly differ with respect to the first step, i.e., methodology used for converting the SNR and SS values of the multiple responses into SNRI and SSI values respectively. In both the methods, PCA is carried out first separately on normalized SNR values and normalized SS values. In PCA-based TOPSIS method (Tong et al., 2004), TOPSIS analysis is used to obtain SNRI and SSI values. These SNRI and SSI values are called as overall performance index (OPI) for SNR (OPI-SNR) and OPI for SS (OPI-SS) respectively. On the other hand, in PCA-based MCE-GRM method (Wang, 2007), multiple criteria evaluation of grey relational model is used to obtain the SNRI and SSI values. The SNRI and SSI values, obtained in MCE-GRM approach, are called as overall relative closeness to ideal solution (RCIS) for SNR (RCIS-SNR) and RCIS for SS (RCIS-SS) respectively. The remaining two steps are the same for both the two methods.

\section{Utility Concept and the Proposed PCA-based utility theory (UT) approach}

\subsection{Utility concept}

Utility can be defined as the usefulness of a product or process in reference to the expectations of the users. The overall usefulness of a product/process can be represented by a unified index, termed as utility which is the sum of individual utilities of various quality characteristics of the product/process. The methodological basis for utility approach is to transform the estimated value of each quality characteristic into a common index.

If $X_{j}$ is the measure of effectiveness of $j^{\text {th }}$ attribute (response variable) and there are $p$ attributes evaluating the outcome space, then the joint utility function (Derek, 1982) can be expressed as:

$$
U\left(X_{1}, X_{2}, \ldots, X_{p}\right)=f\left(U_{1}\left(X_{1}\right), U_{2}\left(X_{2}\right), \ldots, U_{p}\left(X_{p}\right)\right),
$$

where $U_{j}\left(X_{j}\right)$ is the utility of $j^{\text {th }}$ response variable. The overall utility function is the sum of individual utilities if the attributes are independent, and is given as follows:

$$
U\left(X_{1}, X_{2}, \ldots, X_{p}\right)=\sum_{j=1}^{p} U_{j}\left(X_{j}\right)
$$

The attributes may be assigned weights depending upon the relative importance or priorities of the characteristics. The overall utility function after assigning weights to the attributes can be expressed as:

$$
U\left(X_{1}, X_{2}, \ldots, X_{p}\right)=\sum_{j=1}^{p} W_{j} U_{j}\left(X_{j}\right),
$$

where $W_{j}$ is the weight assigned to $j^{\text {th }}$ attribute. The sum of the weights for all the attributes must be equal to 1 .

A preference scale for each response variable is constructed for determining its utility value. Two arbitrary numerical values (preference numbers) 0 and 9 are assigned to the just acceptable and the best 
value of the response variable respectively. The preference number $\left(P_{j}\right)$ for $j^{\text {th }}$ response variable can be expressed on a logarithmic scale as follows (Kumar et al., 2000):

$P_{j}=A_{j} \times \log \left(\frac{X_{j}}{X_{j}^{\prime}}\right)$,

where $X_{j}=$ value of $j^{\text {th }}$ response variable, $X_{j}^{\prime}=$ just acceptable value of $j^{\text {th }}$ response variable and $A_{j}=$ constant for $j^{\text {th }}$ response variable. The value of $A_{j}$ can be found by the condition that if $X_{j}=X_{j}^{B}$ (where $X_{j}^{B}$ is the optimal or best value for $j^{\text {th }}$ response), then $P_{j}=9$. Therefore,

$A_{j}=\frac{9}{\log \left(\frac{X_{j}^{B}}{X_{j}^{\prime}}\right)}$.

The overall utility $(U)$ can be calculated as follows:

$U=\sum_{j=1}^{p} W_{j} P_{j}$

subject to the condition that $\sum_{j=1}^{p} W_{j}=1$.

Let us now consider the application of utility theory for optimizing a multi-response dynamic system. The computed SNR values for $p$ response variables corresponding to $m$ experimental trials can be expressed in the following series:

$$
\mathbf{X}_{1}, \mathbf{X}_{2}, \mathbf{X}_{3}, \ldots, \mathbf{X}_{i}, \ldots, \mathbf{X}_{m},
$$

where

$$
\begin{aligned}
& \mathbf{X}_{1}=\left\{X_{11}, X_{12}, \ldots, X_{1 k}, \ldots, X_{1 p}\right\}, \\
& \mathbf{X}_{i}=\left\{X_{i 1}, X_{i 2}, \ldots, X_{i k}, \ldots, X_{i p}\right\}, \\
& \mathbf{X}_{m}=\left\{X_{m 1}, X_{m 2}, \ldots, X_{m k}, \ldots, X_{m p}\right\} .
\end{aligned}
$$

Here, $\mathbf{X}_{i}$ representing the observed experimental results in $i^{\text {th }}$ trial may be called as the $i^{\text {th }}$ comparative sequence.

Suppose the ideal SNR value of each response variable is known. Then, $\mathbf{x}_{0}=\left\{X_{01}, X_{02}, \ldots, X_{0 k}, \ldots, X_{0 p}\right\}$ may be called as the reference sequence, where $X_{0 j}$ represents the ideal SNR value of $j^{\text {th }}(j=1,2, \ldots, p)$ response variable. It may be noted that $\mathbf{X}_{0}$ and $\mathbf{X}_{i}$ both include $p$ elements, and $X_{0 j}$ and $X_{i j}$ represent the numeric value of $j^{\text {th }}(j=1,2, \ldots, p)$ element in the reference sequence and $i^{\text {th }}$ comparative sequence respectively. So, the amount of deviations in SNR from their ideal values can be estimated for different response variables for the $m$ trials. These differences may be considered as quality losses for SNR for the response variables, which can be appropriately converted to preference numbers and overall utility values for SNR (UV-SNR), using Eqs. (9-11). Then, the process setting that would optimize the UVSNR can be selected examining the level averages of the control factors on the UV-SNR.

Similarly, based on the ideal sequence and comparative sequences for the SS values, quality losses for SS for different response variables can be estimated, which can be appropriately converted to overall utility values for SS (UV-SS). Then, the factors which have significant impact on UV-SS can be identified examining the factor effects on UV-SS and the existence of adjustment factor(s) in the dynamic system can be detected. The level of the adjustment factor may be changed so that the actual output value becomes closer to the target value. 
This approach should work well if the response variables are independent. However, in reality often the multiple responses are correlated. This problem can be overcome by defining the reference and comparative sequences with respect to the principal component scores (PCS) instead of the original response variables. This is because the principal components will be independent even when the original response variables are correlated.

Based on the above logic, PCA-based UT approach is proposed for optimization of multiple responses in a dynamic system.

\subsection{Proposed PCA-based UT Approach}

The computational requirements in the proposed PCA-based UT method can be expressed in the following ten steps:

Step 1: Calculate SNR and SS values corresponding to different trials for each response variable using Eq. 4 and Eq. 5 respectively.

Step 2: Normalize the SNR and SS values for each response variable using the following equations:

$$
\begin{aligned}
& N \eta_{i j}=\frac{\eta_{i j}-\bar{\eta}_{j}}{s d\left(\eta_{j}\right)}, \\
& N S S_{i j}=\frac{S S_{i j}-\overline{S S_{j}}}{s d\left(S S_{j}\right)},
\end{aligned}
$$

where $N \eta_{i j}$ and $N S S_{i j}$ are normalized SNR and SS values respectively for $j^{\text {th }}(j=1,2, \ldots, p)$ response variable in $i^{\text {th }}$ trial, $\bar{\eta}_{j}$ and $\overline{S S_{j}}$ are average SNR and SS values respectively for $j^{\text {th }}(j=1,2, \ldots, p)$ response variable, and $s d\left(\eta_{j}\right)$ and $s d\left(S S_{j}\right)$ are standard deviation of SNR and SS values respectively for $j^{\text {th }}(j=$ $1,2, \ldots, p)$ response variable.

Step 3: Find out reference sequences for the SNR values as well as SS values.

Higher SNR as well as SS values imply better quality. So the elements in reference sequence for SNR will be the largest normalized SNR values for the response variables. Similarly, the elements in reference sequence for SS will be the largest normalized SS values for the response variables.

Step 4: Conduct PCA separately on the normalized SNR values and SS values, and obtain the eigenvalues, eigenvectors and proportion of variation explained by different principal components of normalized SNR and SS values.

Step 5: Compute principal component score (PCS), i.e. the values of each principal component of SNRs for different comparative sequences (trials) and for the reference sequence. Also PCS values of each principal component of SSs for different comparative sequences (trials) and for the reference sequence.

The PCS value of $l^{\text {th }}$ principal component of SNRs corresponding to $i^{\text {th }}$ comparative sequence of SNR ( $P C S_{i l}^{S N R}$ ) can be obtained using Eq. (14) and the value of $l^{\text {th }}$ principal component of the reference sequence can be estimated using Eq. (15) given below:

$$
\begin{aligned}
& P C S_{i l}^{S N R}=a_{l 1} N \eta_{i 1}+a_{l 2} N \eta_{i 2}+\ldots+a_{l p} N \eta_{i p} \quad(i=1,2, \ldots, m \text { and } \quad l=1,2, \ldots, p) \\
& P C S_{0 l}^{S N R}=a_{l 1} \times N \eta_{1}^{\max }+a_{l 2} \times N \eta_{2}^{\max }+\ldots+a_{l p} \times N \eta_{p}^{\max } \quad(l=1,2, \ldots, p)
\end{aligned}
$$

where, $a_{l 1}, a_{l 2}, \ldots, a_{l p}$ are eigen vector of the $l^{\text {th }}$ principal component of SNRs. 
On the other hand, the PCS value of $l^{\text {th }}$ principal component of SS corresponding to $i^{\text {th }}$ comparative sequence of SS ( $P C S_{i l}^{S S}$ ) can be obtained using Eq. (16) and the PCS value of $l^{\text {th }}$ principal component of the reference sequence can be estimated using Eq. (17) given below:

$$
\begin{aligned}
& P C S_{i l}^{S S}=b_{l 1} N S S_{i 1}+b_{l 2} N S S_{i 2}+\ldots+b_{l p} N S S_{i p} \quad(i=1,2, \ldots, m \text { and } \quad l=1,2, \ldots, p) \\
& P C S_{0 l}^{S S}=b_{l 1} \times N S S_{1}^{\max }+b_{l 2} \times N S S_{2}^{\max }+\ldots+b_{l p} \times N S S_{p}^{\max } \quad(l=1,2, \ldots, p)
\end{aligned}
$$

where, $b_{l 1}, b_{l 2}, \ldots, b_{l p}$ are eigen vector of the $l^{\text {th }}$ principal component of SSs.

Step 6: Compute the quality losses in different trials with respect to different principal components.

The absolute difference between $P C S_{i l}^{S N R}$ and $P C S_{0 l}^{S N R}$ values can be considered as the quality loss of SNR for $l^{\text {th }}$ principal component in $i^{\text {th }}$ trial. Similarly, the absolute difference between $P C S_{i l}^{S S}$ and $P C S_{0 l}^{S S}$ values can be considered as the quality loss of SS for $l^{\text {th }}$ principal component in $i^{\text {th }}$ trial. Therefore, the quality losses of SNR and SS for $l^{t h}$ principal component in $i^{\text {th }} \operatorname{trial}\left(L_{i l}^{S S R}\right.$ and $\left.L_{i l}^{S S}\right)$ can be estimated using Eq. (18) and Eq. (19) respectively.

$$
\begin{aligned}
& L_{i l}^{S N R}=\left|P C S_{i l}^{S N R}-P C S_{0 l}^{S N R}\right| \\
& L_{i l}^{S S}=\left|P C S_{i l}^{S S}-P C S_{0 l}^{S S}\right|
\end{aligned}
$$

Step 7: Apply UT for estimating the overall utility values for different trials.

Using Eq. (9) and Eq. (10), the estimated quality losses of SNR for different principal components can be appropriately converted to preference numbers. Then, the overall utility values of SNR (UV-SNR) for different trials can be estimated using En. (11). Similarly, the overall utility values of SS (UV-SS) for different trials can be estimated using Eqs. (9-11). It is suggested here to consider the proportion of variation expressed by different principal components as their weights.

Step 8: Perform ANOVA (analysis of variance) on UV-SNR values and UV-SS values for identification of the most influencing control factors on UV-SNR and UV-SS respectively.

Step 9: Use arithmetic average to calculate the factor effects on UV-SNR and UV-SS values.

Step 10: Determine the optimal factor level combination by higher-the-better factor effects on UV-SNR value.

Step 11: Identify the adjustment factor (a factor significantly affecting UV-SS value but insignificantly affecting UV-SNR value), if any. Then change the level of the adjustment factor in the optimal solution in such a way that the actual output value becomes closer to the target value. Implement the adjusted optimal solution.

\section{Analysis, Results and Discussion}

For the purpose of illustration of the proposed PCA-based UT approach and comparison of its optimization performance with the other available PCA-based approaches, two sets of the past experimental data are taken into consideration. These two data sets are analyzed using the proposed PCA-based UT method, PCA-based TOPSIS method and PCA-based MCE-GRM methods as two 
separate case studies. According to Taguchi, higher SNR implies better quality. Therefore, it is decided to consider the expected total SNR of the response variables at the optimal process condition as the performance metric for comparison of the optimization performance of these three PCA-based approaches.

\subsection{Case study 1}

Hsieh et al. (2005) introduced a problem of the control of two responses relating to optically pure compound performance using eight chemical factors: type of cap, shaking rate, glucose concentration, yeast addition, concentration of enzyme inhibitor, $\mathrm{pH}$ of reaction solution, buffer concentration, and yeast preculture time (denoted as A, B, C, D, E, F, G, and $\mathrm{H}$ respectively). The two optimized responses are $\mathrm{S}-\mathrm{CHBE}\left(Y_{S}\right)$, where a larger response is desired, and R-CHBE $\left(Y_{R}\right)$, where a smaller response is desired. Response $\mathrm{S}-\mathrm{CHBE}\left(Y_{S}\right)$ is more important than R-CHBE $\left(Y_{R}\right)$. When carefully controlled, the S-CHBE forming enzymes are more active than R-CHBE and ultimately produce a higher optical purity. Since altering the substrate concentration would affect both the responses $Y_{S}$ and $Y_{R}$, the substrate concentration was considered as a signal factor $(M)$ in the experiment. Additionally, the freshness of the yeast was considered as a noise factor $(N)$. The $L_{18}$ orthogonal array was employed in that experiment. Six observations were made for both $Y_{S}$ and $Y_{R}$ under each experimental combination. According to the ideal function as given in Eq. (1), the regression models for $Y_{S}$ and $Y_{R}$ on the signal factor $M$ for each experimental run were established and then, SNR and SS for each response were computed using Eq. (4) and Eq. (5) respectively. These computed values are displayed in Table 1. The same experimental data are reanalyzed here using the proposed PCA-based UT approach and the other PCA-based procedures as case study 1.

Higher SNR as well as SS values imply better quality and so the elements in reference sequence for SNR as well as SS should be the largest normalized SNR and SS values for the response variables. Thus, the reference sequence for SNR and SS values are $\{2.141,2.091\}$ and $\{1.826,2.032\}$ respectively. Now, the SNR and SS values of the response variables for the 18 trials are subjected to PCA in STATISTICA software separately. The eigenvalues, proportion of variation explained by different principal components and eigenvectors corresponding to different principal components arising from PCA of SNR and SS values are shown in Tables 2 and 3 respectively. Then applying step 5 described in section 3.2, PCSs for different comparative sequences (i.e. trials) and the reference sequence are computed, and using step 6, the quality losses of each principal component are estimated for different trials. Utility theory is now applied to the dataset of quality losses. Applying Eq. (9) and Eq. (10), the quality losses for each principal component of SNR corresponding to different trials are converted to preference numbers between 0 and 9 . The average preference number for a trial is taken as the measure of overall utility value for SNR (UV-SNR) for that trial. Similarly, overall utility values for SS (UV-SS) for different trials are obtained. On the other hand, overall OPI-SNR and OPI-SS are computed from the same data set applying PCA-based TOPSIS method, and RCIS-SNR and RCIS-SS are computed using PCA-based MCE-GRM method. The computed UV-SNR, UV-SS, OPI-SNR, OPISS, RCIS-SNR and RCIS-SS values for different trials are shown in Table 4.

The ANOVA is carried out separately on UV-SNR, UV-SS, OPI-SNR, OPI-SS, RCIS-SNR and RCISSS values. In these analyses, the F-values for various factors are first computed using the error variance and then, the sum of squares of the factors having F-values less than equal to 1 are pooled with the estimated error variance. The F-values for the remaining factors are finally estimated using the pooled error variance. Table 5 shows the results of these ANOVA. It can be noted from Table 5 that factors B, $\mathrm{D}$ and $\mathrm{E}$ significantly affect the SNRI values (i.e. UV-SNR, OPI-SNR and RCIS-SNR) obtained by all the three PCA-based approaches. However, the factors affecting the SSI (i.e. UV-SS, OPI-SS and RCIS-SS) are different in the three PCA-based approaches. Factor H has significant effect on UV-SS, factors A, D, E and $\mathrm{H}$ have significant effects on OPI-SS and factors A and D have significant effects on RCIS-SS values. It may be recalled that a factor that has significant effect on SSI but no effect on SNRI may be considered as an adjustment factor. This implies that $\mathrm{H}$ is the adjustment factor according 
to the proposed PCA-based UT approach whereas A and $\mathrm{H}$ are adjustment factors according to PCAbased TOPSIS method and A is the adjustment factor according to the PCA-based MCE-GRM method.

The level averages on UV-SNR, UV-SS, OPI-SNR, OPI-SS, RCIS-SNR and RCIS-SS values are displayed in Table 6. Higher UV-SNR, OPI-SNR and RCIS-SNR value imply better quality and therefore, examining Table 6, the optimal solutions based on the proposed PCA-based UT method, PCA-based TOPSIS and PCA-based MCE-GRM method are chosen as $\mathrm{A}_{1} \mathrm{~B}_{3} \mathrm{C}_{1} \mathrm{D}_{3} \mathrm{E}_{2} \mathrm{~F}_{2} \mathrm{G}_{3} \mathrm{H}_{2}$, $\mathrm{A}_{1} \mathrm{~B}_{3} \mathrm{C}_{2} \mathrm{D}_{3} \mathrm{E}_{2} \mathrm{~F}_{1} \mathrm{G}_{3} \mathrm{H}_{1}$ and $\mathrm{A}_{1} \mathrm{~B}_{3} \mathrm{C}_{3} \mathrm{D}_{1} \mathrm{E}_{3} \mathrm{~F}_{3} \mathrm{G}_{3} \mathrm{H}_{1}$, respectively.

As mentioned earlier, the ultimate interest of the process engineer is to maximize the total SNR value. So the SNR values of the individual response variables at different optimal process conditions derived by these methods are predicted using additive model. Table 7 displays the predicted SNR values for the response variables at the different optimal conditions. Examining the results in Table 7, it is found that the optimal condition derived by application of the proposed PCA-based UT method results in higher total SNR, which implies better optimization performance.

\section{Table 1}

Experimental layout and estimates of $\beta, \sigma^{2}, \mathrm{SNR}$ and SS for the responses (case study 1)

\begin{tabular}{|c|c|c|c|c|c|c|c|c|c|c|c|c|c|c|c|c|c|c|c|c|}
\hline \multirow[b]{3}{*}{ Trial } & \multirow{2}{*}{\multicolumn{8}{|c|}{$\begin{array}{l}\text { Experimental layout } \\
\text { Factors and their levels }\end{array}$}} & \multicolumn{4}{|c|}{ Estimates from regression models } & \multirow{2}{*}{\multicolumn{2}{|c|}{ SNR }} & \multirow{2}{*}{\multicolumn{2}{|c|}{ SS }} & \multirow{2}{*}{\multicolumn{2}{|c|}{$\begin{array}{l}\text { Normalized } \\
\text { SNR }\end{array}$}} & \multirow{2}{*}{\multicolumn{2}{|c|}{$\begin{array}{l}\text { Normalized } \\
\text { SS }\end{array}$}} \\
\hline & & & & & & & & & \multicolumn{2}{|c|}{$\beta$} & \multicolumn{2}{|c|}{$\sigma^{2}$} & & & & & & & & \\
\hline & A & B & $\mathrm{C}$ & D & $\mathrm{E}$ & F & $\mathrm{G}$ & $\mathrm{H}$ & $\beta_{Y_{S}}$ & $\beta_{Y_{R}}$ & $\sigma_{Y_{S}}^{2}$ & $\sigma_{Y_{R}}^{2}$ & $Y_{S}$ & $Y_{R}$ & $Y_{S}$ & $Y_{R}$ & $Y_{S}$ & $Y_{R}$ & $Y_{S}$ & $Y_{R}$ \\
\hline 2 & 1 & 1 & 2 & 2 & 2 & 2 & 2 & 2 & 0.4224 & 0.1218 & 0.0468 & 0.0110 & 5.81 & 1.30 & -7.49 & -18.29 & 1.057 & 1.281 & 0.280 & 0.668 \\
\hline 3 & 1 & 1 & 3 & 3 & 3 & 3 & 3 & 3 & 0.4077 & 0.1123 & 0.0701 & 0.0111 & 3.75 & 0.55 & -7.79 & -18.99 & 0.081 & 1.172 & -0.017 & 0.349 \\
\hline 4 & 1 & 2 & 1 & 1 & 2 & 2 & 3 & 3 & 0.4608 & 0.1083 & 0.1156 & 0.0103 & 2.64 & 0.56 & -6.73 & -19.31 & -0.444 & 1.173 & 1.010 & 0.206 \\
\hline 5 & 1 & 2 & 2 & 2 & 3 & 3 & 1 & 1 & 0.4547 & 0.0972 & 0.1376 & 0.0033 & 1.77 & 4.57 & -6.85 & -20.25 & -0.857 & 1.762 & 0.898 & -0.219 \\
\hline 7 & 1 & 3 & 1 & 2 & 1 & 3 & 2 & 3 & 0.3963 & 0.1269 & 0.0633 & 0.0648 & 3.95 & -6.05 & -8.04 & -17.93 & 0.174 & 0.202 & -0.255 & 0.829 \\
\hline 8 & 1 & 3 & 2 & 3 & 2 & 1 & 3 & 1 & 0.3946 & 0.1061 & 0.0241 & 0.0068 & 8.10 & 2.19 & -8.08 & -19.49 & 2.141 & 1.412 & -0.291 & 0.126 \\
\hline 9 & 1 & 3 & 3 & 1 & 3 & 2 & 1 & 2 & 0.5079 & 0.0736 & 0.1013 & 0.0013 & 4.06 & 6.20 & -5.88 & -22.66 & 0.227 & 2.001 & 1.826 & -1.312 \\
\hline 10 & 2 & 1 & 1 & 3 & 3 & 2 & 2 & 1 & 0.4046 & 0.1061 & 0.0665 & 0.0044 & 3.91 & 4.08 & -7.86 & -19.49 & 0.158 & 1.690 & -0.081 & 0.126 \\
\hline 11 & 2 & 1 & 2 & 1 & 1 & 3 & 3 & 2 & 0.3995 & 0.0682 & 0.1717 & 0.0257 & -0.32 & -7.42 & -7.97 & -23.32 & -1.844 & 0.000 & -0.187 & -1.612 \\
\hline 12 & 2 & 1 & 3 & 2 & 2 & 1 & 1 & 3 & 0.3613 & 0.108 & 0.0966 & 0.0239 & 1.31 & -3.12 & -8.84 & -19.33 & -1.075 & 0.633 & -1.030 & 0.195 \\
\hline 13 & 2 & 2 & 1 & 2 & 3 & 1 & 3 & 2 & 0.4377 & 0.1027 & 0.076 & 0.0022 & 4.02 & 6.81 & -7.18 & -19.77 & 0.207 & 2.091 & 0.578 & -0.002 \\
\hline 14 & 2 & 2 & 2 & 3 & 1 & 2 & 1 & 3 & 0.3147 & 0.1723 & 0.0650 & 0.0513 & 1.83 & -2.38 & -10.04 & -15.27 & -0.828 & 0.742 & -2.188 & 2.032 \\
\hline 15 & 2 & 2 & 3 & 1 & 2 & 3 & 2 & 1 & 0.4688 & 0.0809 & 0.1213 & 0.0027 & 2.58 & 3.85 & -6.58 & -21.84 & -0.472 & 1.655 & 1.154 & -0.941 \\
\hline 17 & 2 & 3 & 2 & 1 & 3 & 1 & 2 & 3 & 0.3679 & 0.0595 & 0.0562 & 0.0020 & 3.82 & 2.48 & -8.69 & -24.51 & 0.113 & 1.455 & -0.878 & -2.148 \\
\hline 18 & 2 & 3 & 3 & 2 & 1 & 2 & 3 & 1 & 0.4274 & 0.1043 & 0.0509 & 0.0098 & 5.55 & 0.45 & -7.38 & -19.63 & 0.933 & 1.157 & 0.379 & 0.058 \\
\hline & & & & & & & & & & Iaximum & & & & & & & 2.141 & 2.091 & 1.826 & 2.032 \\
\hline
\end{tabular}

Table 2

Results of PCA on SNR values of the responses (case study 1)

\begin{tabular}{ccccc}
\hline Principal component & Eigen value & Proportion of explained variation & \multicolumn{2}{c}{ Eigenvector } \\
\hline First & 1.381 & 0.69 & 0.707 & 0.707 \\
Second & 0.619 & 0.31 & 0.707 & -0.707 \\
\hline
\end{tabular}

Table 3

Results of PCA on SS values of the responses (case study 1)

\begin{tabular}{ccccc}
\hline Principal component & Eigen value & Proportion of explained variation & \multicolumn{2}{c}{ Eigenvector } \\
\hline First & 1.429 & 0.71 & 0.707 & 0.707 \\
Second & 0.571 & 0.29 & -0.707 & 0.707 \\
\hline
\end{tabular}


Table 4

UV-SNR, UV-SS, OPI-SNR, OPI-SS, RCIS-SNR and RCIS-SS values (case study 1)

\begin{tabular}{ccccccc}
\hline $\begin{array}{c}\text { Trial } \\
\text { no. }\end{array}$ & $\begin{array}{c}\text { Proposed PCA-based UT method } \\
\text { UV-SNR }\end{array}$ & UV-SS & PCA-based TOPSIS method & \multicolumn{2}{c}{ PCA-based MCE-GRM method } \\
\hline 1 & 0.863 & 3.155 & 0.300 & 0.708 & 0.677 & 1.866 \\
2 & 5.102 & 4.268 & 0.787 & 0.565 & 1.766 & 1.592 \\
3 & 2.692 & 3.835 & 0.604 & 0.554 & 1.192 & 1.364 \\
4 & 1.933 & 3.441 & 0.515 & 0.709 & 0.977 & 2.013 \\
5 & 1.707 & 2.774 & 0.541 & 0.740 & 0.815 & 1.829 \\
6 & 3.107 & 2.477 & 0.649 & 0.387 & 1.307 & 1.163 \\
7 & 4.076 & 2.947 & 0.458 & 0.477 & 1.225 & 1.317 \\
8 & 7.297 & 3.308 & 0.984 & 0.535 & 2.899 & 1.206 \\
9 & 3.446 & 2.051 & 0.713 & 0.945 & 1.369 & 2.560 \\
10 & 3.065 & 8.016 & 0.685 & 0.565 & 1.294 & 1.303 \\
11 & 0.271 & 1.096 & 0.177 & 0.690 & 0.472 & 1.071 \\
12 & 1.074 & 1.895 & 0.318 & 0.420 & 0.731 & 0.928 \\
13 & 3.498 & 3.017 & 0.712 & 0.672 & 1.362 & 1.649 \\
14 & 1.362 & 1.446 & 0.376 & 0.197 & 0.813 & 0.699 \\
15 & 2.115 & 2.057 & 0.584 & 0.845 & 0.986 & 1.935 \\
16 & 5.234 & 1.540 & 0.809 & 0.354 & 2.034 & 0.833 \\
17 & 2.851 & 0.633 & 0.652 & 0.627 & 1.241 & 0.686 \\
18 & 4.753 & 3.160 & 0.745 & 0.638 & 1.658 & 1.537 \\
\hline
\end{tabular}

Table 5

Results of ANOVA on UV-SRN and UV-SS, OPI-SNR, OPI-SS, RCIS-SNR and RCIS-SS (case study 1)

\begin{tabular}{|c|c|c|c|c|c|c|c|c|c|c|c|c|c|c|c|c|c|c|}
\hline \multirow{2}{*}{ Source } & \multicolumn{3}{|c|}{ UV-SNR } & \multicolumn{3}{|c|}{ UV-SS } & \multicolumn{3}{|c|}{ OPI-SNR } & \multicolumn{3}{|c|}{ OPI-SS } & \multicolumn{3}{|c|}{ RCIS-SNR } & \multicolumn{3}{|c|}{ RCIS-SS } \\
\hline & SS & DF & $\mathrm{F}$ & SS & DF & $\mathrm{F}$ & SS & DF & $F$ & SS & DF & $\mathrm{F}$ & SS & $\mathrm{DF}$ & $F$ & SS & $\mathrm{DF}$ & $\mathrm{F}$ \\
\hline A & 2.00 & 1 & 3.95 & 0.65 & 1 & - & 0.013 & 1 & - & 0.020 & 1 & 16.31 & 0.148 & 1 & 3.52 & 1.013 & 1 & 19.0 \\
\hline B & 22.6 & 2 & 22.3 & 7.04 & 2 & 5.2 & 0.191 & 2 & 10.5 & 0.000 & 2 & - & 1.990 & 2 & 23.5 & 0.148 & 2 & 1.40 \\
\hline $\mathrm{C}$ & 0.23 & 2 & - & 6.76 & 2 & 5.0 & 0.001 & 2 & - & 0.016 & 2 & 6.53 & 0.048 & 2 & - & 0.535 & 2 & 5.02 \\
\hline D & 11.6 & 2 & 11.5 & 5.85 & 2 & 4.3 & 0.113 & 2 & 6.22 & 0.311 & 2 & 122.1 & 1.214 & 2 & 14.3 & 1.086 & 2 & 10.1 \\
\hline $\mathrm{E}$ & 5.97 & 2 & 5.90 & 1.11 & 2 & - & 0.173 & 2 & 9.48 & 0.087 & 2 & 34.47 & 0.902 & 2 & 10.6 & 0.251 & 2 & 2.36 \\
\hline $\mathrm{F}$ & 1.13 & 2 & - & 7.14 & 2 & 5.2 & 0.036 & 2 & 2.01 & 0.009 & 2 & 3.73 & 0.204 & 2 & 2.41 & 0.412 & 2 & 3.87 \\
\hline $\mathrm{G}$ & 4.98 & 2 & 4.92 & 4.90 & 2 & 3.6 & 0.058 & 2 & 3.19 & 0.017 & 2 & 6.73 & 0.386 & 2 & 4.57 & 0.069 & 2 & - \\
\hline $\mathrm{H}$ & 4.39 & 2 & 4.34 & 9.38 & 2 & 6.9 & 0.094 & 2 & 5.16 & 0.092 & 2 & 36.34 & 0.508 & 2 & 6.02 & 0.624 & 2 & 5.86 \\
\hline Error & 1.67 & 2 & & 1.62 & 2 & & 0.030 & 2 & & 0.004 & 2 & & 0.120 & 2 & & 0.144 & 2 & \\
\hline (P. error) & 3.04 & 6 & & 3.38 & 5 & & 0.045 & 5 & & 0.005 & 4 & & 0.169 & 4 & & 0.213 & 4 & \\
\hline Total & 54.6 & 17 & & 44.4 & 17 & & 0.712 & 17 & & 0.560 & 17 & & 5.525 & 17 & & 4.285 & 17 & \\
\hline
\end{tabular}

Table 6

Level averages on UT-SNR, UT-SS, OPI-SNR, OPI-SS, RCIS-SNR and RCIS-SS (case study 1)

\begin{tabular}{|c|c|c|c|c|c|c|c|c|c|c|c|c|c|c|c|c|c|c|}
\hline \multirow[b]{2}{*}{ Factor } & \multicolumn{3}{|c|}{ UV-SNR } & \multicolumn{3}{|c|}{ UV-SS } & \multicolumn{3}{|c|}{ OPI-SNR } & \multicolumn{3}{|c|}{ OPI-SS } & \multicolumn{3}{|c|}{ RCIS-SNR } & \multicolumn{3}{|c|}{ RCIS-SS } \\
\hline & $\begin{array}{c}\text { Level } \\
1\end{array}$ & $\begin{array}{c}\text { Level } \\
2\end{array}$ & $\begin{array}{c}\text { Level } \\
3\end{array}$ & $\begin{array}{c}\text { Level } \\
1\end{array}$ & $\begin{array}{c}\text { Level } \\
2\end{array}$ & $\begin{array}{c}\text { Level } \\
3\end{array}$ & $\begin{array}{c}\text { Level } \\
1\end{array}$ & $\begin{array}{c}\text { Level } \\
2\end{array}$ & $\begin{array}{c}\text { Level } \\
3\end{array}$ & $\begin{array}{c}\text { Level } \\
1\end{array}$ & $\begin{array}{c}\text { Level } \\
2\end{array}$ & $\begin{array}{c}\text { Level } \\
3\end{array}$ & $\begin{array}{c}\text { Level } \\
1\end{array}$ & $\begin{array}{c}\text { Level } \\
2\end{array}$ & $\begin{array}{c}\text { Level } \\
3\end{array}$ & $\begin{array}{c}\text { Level } \\
1\end{array}$ & $\begin{array}{c}\text { Level } \\
2\end{array}$ & $\begin{array}{c}\text { Level } \\
3\end{array}$ \\
\hline A & 3.358 & 2.692 & & 3.139 & 2.540 & & 1.359 & 1.177 & - & 0.617 & 0.562 & - & 0.624 & 0.556 & - & 1.657 & 1.182 & - \\
\hline B & 2.178 & 2.287 & 4.609 & 3.711 & 2.535 & 2.273 & 1.022 & 1.043 & 1.738 & 0.479 & 0.563 & 0.727 & 0.584 & 0.592 & 0.596 & 1.354 & 1.548 & 1.357 \\
\hline $\mathrm{C}$ & 3.111 & 3.098 & 2.864 & 3.686 & 2.254 & 2.579 & 1.261 & 1.334 & 1.207 & 0.580 & 0.586 & 0.602 & 0.581 & 0.559 & 0.631 & 1.497 & 1.181 & 1.581 \\
\hline $\mathrm{D}$ & 1.913 & 3.368 & 3.793 & 2.072 & 3.010 & 3.437 & 0.954 & 1.259 & 1.590 & 0.490 & 0.594 & 0.685 & 0.754 & 0.585 & 0.432 & 1.689 & 1.475 & 1.095 \\
\hline $\mathrm{E}$ & 2.405 & 3.793 & 2.876 & 2.380 & 2.751 & 3.388 & 1.025 & 1.565 & 1.212 & 0.451 & 0.666 & 0.651 & 0.516 & 0.571 & 0.684 & 1.275 & 1.418 & 1.565 \\
\hline $\mathrm{F}$ & 3.115 & 3.277 & 2.683 & 2.414 & 3.730 & 2.375 & 1.370 & 1.313 & 1.121 & 0.602 & 0.637 & 0.529 & 0.558 & 0.603 & 0.610 & 1.250 & 1.617 & 1.392 \\
\hline $\mathrm{G}$ & 2.281 & 3.386 & 3.407 & 2.144 & 3.400 & 2.976 & 1.073 & 1.303 & 1.427 & 0.509 & 0.636 & 0.623 & 0.561 & 0.578 & 0.633 & 1.452 & 1.333 & 1.473 \\
\hline $\mathrm{H}$ & 3.300 & 3.443 & 2.331 & 3.745 & 2.408 & 2.366 & 1.388 & 1.385 & 1.030 & 0.640 & 0.641 & 0.487 & 0.672 & 0.602 & 0.497 & 1.613 & 1.478 & 1.168 \\
\hline
\end{tabular}

Table 7

Predicted SNR values at the optimal conditions derived by the proposed and other PCA-based methods

\begin{tabular}{lcccc}
\hline \multirow{2}{*}{ Optimization method } & \multirow{2}{*}{ Optimal condition } & \multicolumn{2}{c}{ Predicted SNR } & \multirow{2}{*}{ Total SNR } \\
\cline { 3 - 4 } & & $Y_{S}$ & $Y_{R}$ & \multirow{2}{*}{} \\
Proposed PCA-based UT method & $\mathrm{A}_{1} \mathrm{~B}_{3} \mathrm{C}_{1} \mathrm{D}_{3} \mathrm{E}_{2} \mathrm{~F}_{2} \mathrm{G}_{3} \mathrm{H}_{2}$ & $9.089 \mathrm{~dB}$ & $2.453 \mathrm{~dB}$ & $11.542 \mathrm{~dB}$ \\
PCA-based TOPSIS method & $\mathrm{A}_{1} \mathrm{~B}_{3} \mathrm{C}_{2} \mathrm{D}_{3} \mathrm{E}_{2} \mathrm{~F}_{1} \mathrm{G}_{3} \mathrm{H}_{1}$ & $8.403 \mathrm{~dB}$ & $2.449 \mathrm{~dB}$ & $10.852 \mathrm{~dB}$ \\
PCA-based MCE-GRM method & $\mathrm{A}_{1} \mathrm{~B}_{3} \mathrm{C}_{3} \mathrm{D}_{1} \mathrm{E}_{3} \mathrm{~F}_{3} \mathrm{G}_{3} \mathrm{H}_{1}$ & $4.394 \mathrm{~dB}$ & $4.920 \mathrm{~dB}$ & $9.315 \mathrm{~dB}$ \\
\hline
\end{tabular}




\subsection{Case study 2}

Chang (2006) simulated an example of a dynamic system with multiple responses for illustrating application of their proposed neural network-based desirability function approach for optimizing multiple dynamic responses. That example involved three response variables, i.e. $Y_{1}, Y_{2}$ and $Y_{3}$. Chang (2006) obtained the hypothetical experimental data based on Monte Carlo simulation. Six control factors, i.e. A, B, C, D, E and F were allocated to $L_{18}$ orthogonal array. The signal factor had three levels, e.g. $\mathrm{M}_{1}, \mathrm{M}_{2}$ and $\mathrm{M}_{3}$, and the corresponding values were 10, 20 and 30 respectively. Two levels of noise factor $\left(\mathrm{N}_{1}\right.$ and $\left.\mathrm{N}_{2}\right)$ were also in the system. Twelve observations were simulated for $Y_{1}, Y_{2}$ and $Y_{3}$ under each experimental combination. The simulated experimental data are available in Chang (2006). The same experimental data are reanalyzed here using the proposed PCA-based UT approach and the other PCA-based procedures as case study 2. According to the ideal function as given in Eq. (1), the regression models for $Y_{1}, Y_{2}$ and $Y_{3}$ on the signal factor $M$ for each experimental run were established and then, SNR and SS for each response were computed using Eq. (4) and Eq. (5) respectively. These computed values are displayed in Table 8.

As higher SNR as well as SS values imply better quality, the largest normalized SNR and SS values for the response variables are taken as the elements in the reference sequence for SNR and SS respectively, i.e. the reference sequence for SRN and SS are considered as $\{2.39,2.12,2.41\}$ and $\{1.19,1.46,1.38\}$ respectively. Now, the SNR and SS values of the response variables for the 18 trials are subjected to PCA in STATISTICA software separately. The eigenvalues, proportion of variation explained by different principal components and eigenvectors corresponding to different principal components arising from PCA of SNR and SS values are shown in Tables 9 and 10 respectively. Then applying steps 5-6 described in Section 3.2, PCSs for different comparative sequences (i.e. trials), PCSs for the reference sequence and the quality losses of each principal component are estimated for different trials. Utility theory (UT) is then applied to obtain the UV-SNR and US-SS values for different trials. On the other hand, overall OPI-SNR and OPI-SS are computed from the same data set applying PCA-based TOPSIS method, and RCIS-SNR and RCIS-SS are computed using PCA-based MCE-GRM method. The computed UV-SNR, UV-SS, OPI-SNR, OPI-SS, RCIS-SNR and RCIS-SS values for different trials are shown in Table 11.

The ANOVA is carried out separately on UV-SNR, UV-SS, OPI-SNR, OPI-SS, RCIS-SNR and RCISSS values. Table 12 shows the results of these ANOVA. It can be noted from Table 12 that only factors F significantly affect UV-SNR and OPI-SNR whereas only factor E significantly affect RCIS-SNR. On the other hand, examining the ANOVA of SSI values (i.e. UV-SS, OPI-SS and RCIS-SS) it is found that, there is no adjustment factor according to the proposed PCA-based UT approach whereas B, C and $\mathrm{E}$ are the adjustment factors according to PCA-based TOPSIS method and B, C, D and E are the adjustment factor according to the PCA-based MCE-GRA method.

The level averages on UV-SNR, UV-SS, OPI-SNR, OPI-SS, RCIS-SNR and RCIS-SS values are displayed in Table 13. Higher UV-SNR, OPI-SNR and RCIS-SNR value imply better quality and therefore, examining Table 13, the optimal solutions based on the proposed PCA-based UT method, PCA-based TOPSIS and PCA-based MCE-GRA method are chosen as $\mathrm{A}_{3} \mathrm{~B}_{1} \mathrm{C}_{3} \mathrm{D}_{3} \mathrm{E}_{3} \mathrm{~F}_{3}, \mathrm{~A}_{1} \mathrm{~B}_{3} \mathrm{C}_{1} \mathrm{D}_{3} \mathrm{E}_{2} \mathrm{~F}_{3}$ and $\mathrm{A}_{3} \mathrm{~B}_{3} \mathrm{C}_{3} \mathrm{D}_{3} \mathrm{E}_{2} \mathrm{~F}_{3}$, respectively.

As mentioned earlier, the ultimate interest of the process engineer is to maximize the total SNR value. So the SNR values of the individual response variables at different optimal process conditions derived by these methods are predicted using additive model (Taguchi, 1990). Table 14 displays the predicted SNR values for the response variables at the different optimal conditions. Examining the results in Table 14, it is found that the optimal condition derived by application of the proposed PCA-based UT method results in higher total SNR, which implies better optimization performance. 
Table 8

Experimental layout and estimates of $\beta, \sigma^{2}, \mathrm{SNR}$ and SS for the responses (case study 2)

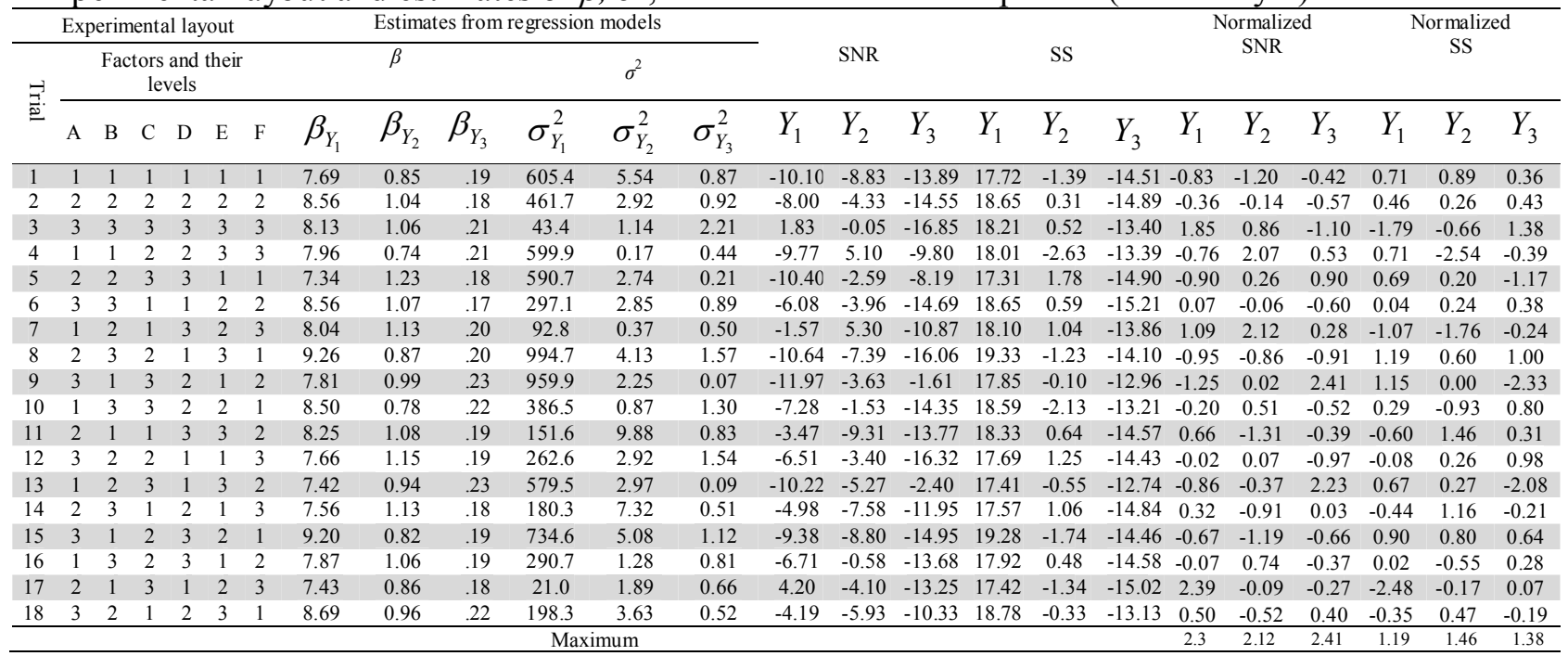

Table 9

Results of PCA of normalized SN ratios for the responses (case study 2)

\begin{tabular}{lccccc}
\hline Principal component & Eigen value & Proportion of explained variation & \multicolumn{3}{c}{ Eigenvector } \\
\hline First & 1.394 & 0.46 & 0.728 & 0.146 & -0.669 \\
Second & 1.095 & 0.36 & 0.187 & 0.897 & 0.400 \\
Third & 0.511 & 0.17 & 0.659 & -0.417 & 0.626 \\
\hline
\end{tabular}

Table 10

Results of PCA of normalized SS for the responses (case study 2)

\begin{tabular}{cccccc}
\hline Principal component & Eigen value & Proportion of explained variation & \multicolumn{3}{c}{ Eigenvector } \\
\hline First & 1.505 & 0.50 & 0.496 & -0.691 & 0.525 \\
Second & 0.957 & 0.32 & 0.731 & 0.006 & -0.682 \\
Third & 0.538 & 0.18 & 0.468 & 0.723 & 0.508 \\
\hline
\end{tabular}

Table 11

UV-SNR, UV-SS, OPI-SNR, OPI-SS, RCIS-SNR and RCIS-SS values (case study 2)

\begin{tabular}{ccccccc}
\hline \multirow{2}{*}{ Trial no. } & Proposed PCA-based UT method & \multicolumn{2}{c}{ PCA-based TOPSIS method } & \multicolumn{2}{c}{ PCA-based MCE-GRM method } \\
\cline { 2 - 7 } & UV-SNR & UV-SS & OPI-SNR & OPI-SS & RCIS-SNR & RCIS-SS \\
\hline 1 & 1.291 & 5.702 & 0.351 & 0.502 & 0.707 & 1.185 \\
2 & 2.360 & 3.594 & 0.467 & 0.537 & 0.948 & 1.175 \\
3 & 5.640 & 1.193 & 0.767 & 0.382 & 1.074 & 0.827 \\
4 & 5.942 & 0.933 & 0.558 & 0.670 & 0.644 & 0.580 \\
5 & 3.611 & 4.050 & 0.358 & 0.000 & 1.005 & 1.178 \\
6 & 2.646 & 3.301 & 0.524 & 0.511 & 1.057 & 0.755 \\
7 & 6.701 & 0.769 & 0.767 & 0.302 & 0.788 & 1.862 \\
8 & 1.083 & 5.943 & 0.369 & 0.790 & 1.142 & 0.824 \\
9 & 3.129 & 3.546 & 0.281 & 0.359 & 0.929 & 1.748 \\
10 & 3.448 & 2.406 & 0.547 & 0.751 & 0.734 & 0.949 \\
11 & 2.018 & 4.059 & 0.459 & 0.416 & 0.723 & 0.674 \\
12 & 2.473 & 3.470 & 0.547 & 0.167 & 0.770 & 0.740 \\
13 & 3.607 & 3.038 & 0.259 & 0.307 & 1.096 & 0.688 \\
14 & 2.539 & 5.555 & 0.432 & 0.158 & 0.902 & 2.131 \\
15 & 1.112 & 5.462 & 0.360 & 0.845 & 0.862 \\
16 & 4.100 & 2.252 & 0.575 & 0.323 & 1.212 & 1.161 \\
\hline 17 & 5.345 & 1.080 & 0.669 & 0.408 & 0.598 & 1.132 \\
\hline
\end{tabular}


Table 12

ANOVA results on UV-SRN, UV-SS, OPI-SNR, OPI-SS, RCIS-SNR and RCIS-SS (case study 2)

\begin{tabular}{|c|c|c|c|c|c|c|c|c|c|c|c|c|c|c|c|c|c|c|}
\hline \multirow{2}{*}{ Source } & \multicolumn{3}{|c|}{ UV-SNR } & \multicolumn{3}{|c|}{ UV-SS } & \multicolumn{3}{|c|}{ OPI-SNR } & \multicolumn{3}{|c|}{ OPI-SS } & \multicolumn{3}{|c|}{ RCIS-SNR } & \multicolumn{3}{|c|}{ RCIS-SS } \\
\hline & SS & DF & $\mathrm{F}$ & & & & SS & $\mathrm{DF}$ & $\mathrm{F}$ & SS & DF & $\mathrm{F}$ & SS & $\mathrm{DF}$ & $\mathrm{F}$ & SS & DF & $\mathrm{F}$ \\
\hline A & 6.2321 & 2 & 2.30 & 7.0900 & 2 & 2.56 & 0.0078 & 2 & - & 0.0337 & 2 & - & 0.0222 & 2 & - & 0.0243 & 2 & - \\
\hline B & 1.0889 & 2 & - & 0.5920 & 2 & - & 0.0248 & 2 & - & 0.1528 & 2 & $7.12^{*}$ & 0.0521 & 2 & 1.81 & 0.7291 & 2 & $39.75^{*}$ \\
\hline $\mathrm{C}$ & 5.5184 & 2 & 2.03 & 5.4930 & 2 & 1.99 & 0.0014 & 2 & - & 0.1144 & 2 & $5.33^{*}$ & 0.0004 & 2 & - & 0.6561 & 2 & $35.77^{*}$ \\
\hline D & 3.9110 & 2 & 1.44 & 1.9248 & 2 & - & 0.0342 & 2 & 1.59 & 0.0538 & 2 & 2.50 & 0.0389 & 2 & - & 0.1052 & 2 & $5.74^{*}$ \\
\hline E & 2.2990 & 2 & - & 5.7002 & 2 & 2.06 & 0.0527 & 2 & 2.45 & 0.3436 & 2 & $15.99^{*}$ & 0.4078 & 2 & $14.16^{*}$ & 1.0034 & 2 & $54.70^{*}$ \\
\hline $\mathrm{F}$ & 19.14 & 2 & $7.06^{*}$ & 16.46 & 2 & $5.95^{*}$ & 0.1710 & 2 & $7.96^{*}$ & 0.1759 & 2 & $8.19^{*}$ & 0.0900 & 2 & 3.12 & 0.9204 & 2 & $50.18^{*}$ \\
\hline Error & 8.8182 & 5 & & 9.9337 & 5 & & 0.0841 & 5 & & 0.0151 & 5 & & 0.0970 & 5 & & 0.0399 & 5 & \\
\hline (P Error) & 12.2061 & 9 & & 12.4504 & 9 & & 0.1181 & 11 & & 0.0488 & 7 & & 0.1584 & 11 & & 0.0642 & 7 & \\
\hline Total & 47.0064 & 17 & & 47.1922 & 17 & & 0.3761 & 17 & & 0.8893 & 17 & & 0.7083 & 17 & & 3.4784 & 17 & \\
\hline
\end{tabular}

Statistically significant at $5 \%$ level

Table 13

Level averages on UV-SRN, UV-SS, OPI-SNR, OPI-SS, RCIS-SNR and RCIS-SS (case study 2)

\begin{tabular}{|c|c|c|c|c|c|c|c|c|c|c|c|c|c|c|c|c|c|c|}
\hline \multirow[t]{3}{*}{ Factor } & \multicolumn{3}{|c|}{ UV-SNR } & \multicolumn{3}{|c|}{ UV-SS } & \multicolumn{3}{|c|}{ OPI-SNR } & \multicolumn{3}{|c|}{ OPI-SS } & \multicolumn{3}{|c|}{ RCIS-SNR } & \multicolumn{3}{|c|}{ RCIS-SS } \\
\hline & Level & Level & Level & Level & Level & Level & Level & Level & Level & Level & Level & Level & Level 1 & Level & Level & Level & Level & Level \\
\hline & 1 & 2 & 3 & 1 & 2 & 3 & 1 & 2 & 3 & 1 & 2 & 3 & & 2 & 3 & 1 & 2 & 3 \\
\hline A & 2.872 & 3.243 & 3.973 & 3.404 & 3.723 & 2.847 & 0.510 & 0.459 & 0.490 & 0.476 & 0.385 & 0.477 & 0.962 & 0.927 & 1.012 & 1.1451 & 1.0600 & 1.1277 \\
\hline B & 4.182 & 2.826 & 3.080 & 2.517 & 4.047 & 3.410 & 0.446 & 0.476 & 0.536 & 0.533 & 0.318 & 0.486 & 0.968 & 0.901 & 1.033 & 1.3049 & 0.8335 & 1.1943 \\
\hline D & 2.741 & 3.483 & 3.864 & 3.756 & 3.254 & 2.964 & 0.453 & 0.457 & 0.548 & 0.447 & 0.512 & 0.378 & 0.906 & 0.975 & 1.019 & 1.1333 & 1.1913 & 1.0081 \\
\hline E & 2.857 & 3.602 & 3.628 & 4.096 & 2.769 & 3.109 & 0.424 & 0.556 & 0.479 & 0.251 & 0.559 & 0.527 & 0.757 & 1.104 & 1.039 & 0.7929 & 1.3581 & 1.1818 \\
\hline $\mathrm{F}$ & 2.337 & 2.977 & 4.773 & 4.509 & 3.298 & 2.167 & 0.407 & 0.428 & 0.623 & 0.581 & 0.409 & 0.348 & 0.963 & 0.883 & 1.056 & 1.4307 & 0.9548 & 0.9473 \\
\hline
\end{tabular}

Table 14

Predicted SN ratios at the optimal conditions derived by the three methods

\begin{tabular}{lccccc}
\hline \multirow{2}{*}{ Optimization method } & Optimal & \multicolumn{2}{c}{ Predicted SNR values } & Total \\
\cline { 3 - 5 } & condition & $Y_{1}$ & $Y_{2}$ & $Y_{3}$ & SNR \\
\hline Proposed PCA-based UT method & $\mathrm{A}_{1} \mathrm{~B}_{3} \mathrm{C}_{3} \mathrm{D}_{3} \mathrm{E}_{2} \mathrm{~F}_{3}$ & $-0.256 \mathrm{~dB}$ & $-0.777 \mathrm{~dB}$ & $-10.448 \mathrm{~dB}$ & $-11.481 \mathrm{~dB}$ \\
PCA-based TOPSIS method & $\mathrm{A}_{1} \mathrm{~B}_{3} \mathrm{C}_{1} \mathrm{D}_{3} \mathrm{E}_{2} \mathrm{~F}_{3}$ & $1.257 \mathrm{~dB}$ & $2.680 \mathrm{~dB}$ & $-17.591 \mathrm{~dB}$ & $-13.654 \mathrm{~dB}$ \\
PCA-based MCE-GRM method & $\mathrm{A}_{3} \mathrm{~B}_{3} \mathrm{C}_{3} \mathrm{D}_{3} \mathrm{E}_{2} \mathrm{~F}_{3}$ & $2.242 \mathrm{~dB}$ & $1.543 \mathrm{~dB}$ & $-16.077 \mathrm{~dB}$ & $-12.293 \mathrm{~dB}$ \\
\hline
\end{tabular}

\section{Conclusion}

Industries are increasingly emphasizing optimization of multiple responses in dynamic system in the light of increasing complexities of modern manufacturing design. Often the multiple responses are correlated. Hence, the PCA-based approaches which take into account the possible correlation among the responses have gained popularity among the practitioners. This paper proposes a new PCA-based approach, called PCA-based utility theory (UT) approach. Two sets of past experimental data are analyzed using the proposed method and two other known PCA-based approaches. The results show that the proposed PCA-based UT approach outperforms the other PCA-based approaches in terms of overall optimization performance. This implies that the proposed PCA-based UT method is very promising for optimization of dynamic systems with multiple responses.

\section{References}

Bae, S.J., \& Tsui, K.L. (2006). Analysis of dynamic robust design experiment with explicit \& hidden noise variables. Quality Technology \& Quantitative Management, 3, 55-75.

Chang, H.H. (2006). Dynamic multi-response experiments by back propagation networks and desirability functions. Journal of the Chinese Institute of Industrial Engineers, 23, 280-288.

Chang, H.H. (2008). A data mining approach to dynamic multiple responses in Taguchi experimental design. Expert System with Applications, 35, 1095-1103.

Chang, H.H., \& Chen, Y.K. (2011). Neuro-genetic approach to optimize parameter design of dynamic multiresponse experiments. Applied Soft Computing, 11, 436 - 442.

Chen, S.P. (2003). Robust design with dynamic characteristics using stochastic sequential quadratic programming. Engineering Optimization, 35, 79-89.

Derek, W.B. (1982). Analysis of Optimal Decisions. New York: John Wiley and Sons.

Derringer, G., \& Suich, R. (1980). Simultaneous optimization of several response variables. Journal of Quality Technology, 12, 214-219. 
Hsieh, K.L., Tong, L.I., Chiu, H.P., \& Yeh, H.Y. (2005). Optimization of a multi-response problem in Taguchi's dynamic system. Computers and Industrial Engineering, 49, 556-571.

Jeong, I.N., \& Kim, K.J. (2009). An interactive desirability function method to multiresponse optimization. European Journal of Operational Research, 195, 412-426.

Joseph, V.R., \& Wu, C.F.J. (2002). Robust parameter design of multiple-target systems. Technometrics, 44, 338-346.

Khuri, A.I., \& Conlon, M. (1981). Simultaneous optimization of multiple responses represented by polynomial regression functions. Technometrics, 23, 363-375.

Kim, K.J. \& Lee, D.K.J. (2006). Optimization of multiple responses considering both location and dispersion effects. European Journal of Operational Research, 169, 133-145.

Kumar, P., Barua, P.B., \& Gaindhar, J.L. (2000). Quality optimisation (multi-characteristics) through Taguchi technique and utility concept. Quality and Reliability Engineering International, Vol. 16, pp. 475-485.

Lesperance, M.L., \& Park, S.M. (2003). GLMs for the analysis of robust designs with dynamic characteristics. Journal of Quality Technology, 35, 253-263.

Liao, H.C. (2006). Multi-response optimization using weighted principal component. International Journal on Advanced Manufacturing Technology, 27, 720-725.

Lunani, M., Nair, V.N., \& Wasserman, G.S. (1997). Graphical methods for robust design with dynamic characteristics. Journal of Quality Technology, 29, 327-338.

McCaskey, S.D., \& Tsui, K.L. (1997). Analysis of dynamic robust design experiments. International Journal of Production Research, 35, 1561-1574.

Miller, A., \& Wu, C.F.J. (1996). Parameter design for signal-response systems: A different look at Taguchi's dynamic parameter design. Statistical Science, 11, 122-136.

Pal, S., \& Gauri, S.K. $\left(2010^{\mathrm{a}}\right)$. Multi-response optimization using multiple regression based weighted signal-tonoise ratio (MRWSN). Quality Engineering, 22, 336-350.

Pal, S., \& Gauri, S.K. $\left(2010^{\mathrm{b}}\right)$. Assessing effectiveness of the various performance metrics for multi-response optimization using multiple regression. Computers and Industrial Engineering, 59, 976-985.

Pignatiello, J.J. (1993). Strategies for robust multi-response quality engineering. Industrial Engineering Research Development-IIET, 25, 5-15.

Su, C.T., \& Tong, L.I. (1997). Multi-response robust design by principal component analysis. Total Quality Management, 8, 409-416.

Su, C.T., Chen, H.L., \& Chan, H.L. (2005). Applying neural network and scatter search to optimize parameter design with dynamic characteristics. Journal of the Operational Research Society, 56, 1132-1140.

Taguchi, G. (1990). Introduction to Quality Engineering. Tokyo, Japan: Asian Productivity Organization.

Tong, L.I., \& Hsieh, K.L. (2001). A novel means of Applying artificial neural networks to optimize multiresponse problem. Quality Engineering, 13, 11-18.

Tong, L.I., Wang, C.H., Houng, J.Y., \& Chen, J.Y. (2002). Optimizing dynamic multiresponse problems using the dual-response-surface method. Quality Engineering, 14, 115-125.

Tong, L.I., Wang, C.H., Chen, C.C., \& Chen, C.T. (2004). Dynamic multiple responses by ideal solution analysis. European Journal of Operational Research, 156, 433-441.

Tong, L.I., Chen, C.C., \& Wang, C.H. (2007). Optimization of multi-response processes using the VIKOR method. International Journal on Advanced Manufacturing Technology, 31, 1049-1057.

Tong, L.I., Wang, C.H., \& Tsai, C.W. (2008). Robust design for multiple dynamic quality characteristics using data envelopment analysis. Quality and Reliability Engineering International, 24, 557-571.

Tsui, K. (1999). Modelling and analysis of dynamic robust design experiments. IIE Transactions, 31, 1113 1122.

Wang, C.H. (2007). Dynamic multi-response optimization using principal component analysis and multiple criteria evaluation of the grey relation model. International Journal on Advanced Manufacturing Technology, 32, 617-624.

Wasserman, G.S. (1996). Parameter design with dynamic characteristics: A regression perspective. Quality and Reliability Engineering International, 12, 113-117.

Wu, C.F.J., \& Hamada, M. (2000). Experiments: Planning, analysis, and parameter design optimization. New York: Wiley-Interscience.

$\mathrm{Wu}$, C.F.J. (2005). Optimization of correlated multiple quality characteristics using desirability function. Quality Engineering, 17, 119-126.

$\mathrm{Wu}$, C.F.J. (2009). Robust design of nonlinear multiple dynamic quality characteristics. Computers and Industrial Engineering, 56, 1328-1332. 